\title{
RETROALIMENTACIÓN EN EDUCACIÓN EN LÍNEA: UNA ESTRATEGIA PARA LA CONSTRUCCIÓN DEL CONOCIMIENTO
}

\author{
(FEEDBACK ON ONLINE EDUCATION: A STRATEGY FOR KNOWLEDGE BUILDING)
}

\author{
Marta Araceli Alvarado García \\ Universidad TecVirtual del Sistema Tecnológico de Monterrey, México
}

\section{RESUMEN}

La sociedad ha pasado por diferentes etapas en el área de la educación a lo largo de la historia, en cada una de ellas se han implementado diversas estrategias, modelos, metodologías, etc, que han cubierto requerimientos en contextos vigentes. Hace solo algunos años se integra a esta evolución la modalidad en línea, que se soporta en las tecnologías y se caracteriza básicamente por romper barreras de tiempo y espacio durante procesos de formación académica. Esta innovadora forma de enseñar y aprender conlleva a reflexionar sobre el proceso de evaluación: ¿deberá requerirse también una nueva forma de evaluar? ¿es la retroalimentación importante en el proceso de evaluación? ¿cómo podría definirse el concepto de "retroalimentación“? ¿una retroalimentación efectiva, eficaz, adecuada y sistemática generará conocimiento en línea? A través de este artículo se describirá el proceso de retroalimentación como detonante para la construcción del conocimiento.

Palabras clave: construcción del conocimiento, retroalimentación, educación en línea.

\begin{abstract}
Within the field of education there have been a number of different stages to describe its evolution over time. For each of these stages, it has been necessary to implement different strategies, models, and methodologies. These have helped to meet the needs of specific requirements within various educational contexts that occur today. Only recently, in the last few years, however, has it been possible to integrate the online modality within the changing landscape of traditional education. This online system is generally supported by new technologies- and is basically characterized by breaking time and space barriers during academic training processes. This innovative way of teaching and learning leads to a deeper reflection on the evaluation process. We are faced with questions like: Is a new form of evaluation required? Is feedback important in the evaluation process? How should the concept of "feedback" be defined? Would effective, efficient, adequate, and systematic feedback generate knowledge online? In this article, we examine the process of feedback as a catalyst for knowledge creation.
\end{abstract}


Keywords: knowledge creation, feedback, online education.

En una sociedad que está requiriendo de perfiles de egresados con habilidades, conocimientos y competencias propias del contexto actual, surge el binomio tecnología/educación para dar paso al concepto de educación en línea.

Este modelo promete ser una solución importante no solo para desarrollar habilidades "adhoc" a nuestra realidad, pues es "La mejor vía para lograr de manera rápida y eficiente el incremento de competencias” CIMED (2005, p. 55), sino también para combatir el rezago educativo de muchos países. Tal como lo señala Quesada (2006, p. 2) "En México la educación a distancia “en línea” se percibe como una de las mejores opciones para ampliar la cobertura educativa en el nivel superior de enseñanza...tanto en instituciones públicas como privadas” y que McAnally y Organista (2007, p. 85) refuerzan al mencionar..."La educación en línea ha generado tantas expectativas que se ha posicionado en el discurso de organismos nacionales e internacionales como uno de los caminos a seguir”.

El mundo contemporáneo exige nuevas formas de vivir y también de actuar, surge la necesidad de una mejor organización de nuestro tiempo y también de seguir con procesos de formación continua. Esta situación es retomada por CIMED al dar a conocer que: "Existe conciencia en el mundo entero de que se aprende durante toda la vida, pero con los cambios que se producen en el entorno, la educación tiende a adecuarse para que pueda aprovecharse mejor...hay raíces que siguen siendo útiles, pero en la superficie afloran las nuevas tecnologías que acompañan a la globalización..." (2005, pp. 56-57).

Esta modalidad innovadora es el resultado de cambios económicos, políticos, culturales y sociales, además del surgimiento y crecimiento vertiginoso de la tecnología. De esta manera, Angulo (2009) referencia que más que ver a las tecnologías como un recurso que apoya a la parte didáctica, se deberá considerar como aquella que brindará la posibilidad de potenciar la capacidad y versatilidad de cualquier institución que promueva el conocimiento.

Es así que se rompen paradigmas importantes referentes a las formas de enseñar y aprender y se hace entonces necesario el desarrollo de habilidades y competencias de profesores y alumnos que coexistirán en el espacio virtual de un curso (Angulo, 2009). Por lo anterior se requiere de formación, entrenamiento y/o capacitación de quienes intervienen en esta modalidad, desde los expertos en el conocimiento, los diseñadores instruccionales, los profesores e incluso los alumnos (García y Montiel, 2008). 
Sin embargo, a pesar de la preocupación de impulsar la educación mediante una modalidad soportada en la tecnología y de alto alcance, pareciera que los procesos de evaluación solo se trasladan de un formato presencial a uno virtual sin evidenciar innovación en su diseño.

Los diferentes tipos de evaluación que ocurren durante el proceso de enseñanza - aprendizaje son básicamente tres: diagnóstica, formativa y sumativa. La formativa cobra un peso mayor debido a que se basa en una continua construcción de conocimiento durante todo el periodo que comprende un curso. Lo anterior a través de una retroalimentación cimentada en la detección oportuna de omisiones, errores y aciertos de los alumnos en las diferentes actividades que realizan. Eso le permite al profesor seguir conduciendo y reconduciendo el aprendizaje de acuerdo a los objetivos que se busca alcanzar y dando a conocer cómo se puede seguir mejorando. La retroalimentación es entonces la parte central de la evaluación formativa por lo que deberá ser constante, clara, oportuna, suficiente y pertinente (Quesada, 2006). Es el medio a través del cual el alumno identifica lo que le hace falta para lograr el éxito de acuerdo a lo que se espera de él.

La retroalimentación deberá darse en ambos sentidos (maestro - alumno, alumno - maestro) y asegurar así que el aprendizaje se está dando. Estos “...procesos de "Feed - Back" adaptan y readaptan de manera progresiva el conocimiento ajustándolo de un modo correcto" (Barberá, 2006, p.11).

Siendo la retroalimentación la columna vertebral en el proceso de construcción del conocimiento; podemos decir que la intervención del profesor para este fin es esencial, tanto en actividades individuales como aquellas que se desarrollan en equipo (foros).

Para que una retroalimentación esté completa debe incluir tres conceptos: "Feed - Up" que se refiere a qué dirección está tomando el alumno, si se está dando cuenta hacia dónde va, es básicamente no perder de vista el objetivo de la actividad que se está revisando y hacer referencia, en síntesis, de la actividad previa para ligar el conocimiento previo con el actual; el "Feed - Forward" que esencialmente y en retroalimentación enviada por su profesor, debe contestar al alumno las interrogantes sobre ¿qué sigue ahora? ¿cómo puede mejorar para la siguiente actividad? Y el "Feed - Back" que le ayuda al alumno a darse cuenta cómo se está desempeñando.

El presente documento se enfocará en la retroalimentación que el profesor debe realizar tanto a actividades individuales como grupales (foros). 
Si se toma en cuenta que la educación en línea es una de las estrategias para proveer de educación a un gran sector de la sociedad, es preciso seguir estudiando y detectando formas para seguir desarrollándola y ofrecer así calidad académica bajo este modelo. La primera reflexión que tendría que hacerse entonces y que tiene que ver directamente con la calidad podría ser ¿qué papel juega la retroalimentación en esta modalidad?

Definir el concepto de retroalimentación es sin duda muy interesante y más adelante se reflejará en este documento, pero antes se puntualizará que para lograr resultados satisfactorios en este proceso de retroalimentación se requieren: habilidades pedagógicas del tutor, alto nivel de actividad de los alumnos y un esfuerzo compartido entre ambas partes (Jackson y Graesser, 2006). La actuación del tutor se resume en compromiso y disciplina, pues su ingreso al curso deberá ser diario y las retroalimentaciones entregadas a los alumnos de acuerdo a las políticas de tiempos de respuesta de la institución. El profesor (tutor) deberá además mantener liderazgo intelectual, motivar al alumno y permanecer en una evaluación constante durante la socialización del conocimiento.

De las variables anteriores se desprenden algunos aspectos a considerar:

El tutor deberá:

1. Poseer habilidades de comunicación. Comunicar con claridad, veracidad, relevancia, calidad, cantidad adecuada y estructura.

2. Dominar el contenido temático y técnica didáctica en la que se apoyará.

3. Informar al alumno de las políticas y lineamientos del curso y de cada actividad al inicio (aun cuando en algún apartado del curso se encontraran).

4. Asegurar claridad en la tarea.

5. Tener conocimientos técnicos básicos indispensables referentes a la plataforma y otras tecnologías utilizadas en el proceso.

6. Ser autorregulado, participativo y generador de pensamiento crítico y reflexivo. 
7. Promover la autonomía en el alumno debido a que él será el protagonista de su aprendizaje y que su autorregulación, autodirección, actitud y nivel de participación serán los que determinen la calidad del mismo.

Es muy importante mencionar que todo lo anterior deberá darse en un clima en el que los valores y la ética prevalezcan.

Jackson y Graesser dan a conocer que "la retroalimentación podría considerarse una especie de andamiaje y por ello ser el paso inicial en dirección hacia una sutil estrategia tutorial" (2006, p. 34), lo cual debiera ser una interesante reflexión para cada profesor.

La comunicación: deber darse de tal forma que le permita al docente conocer a cada alumno y posibilitarle encontrar motivadores individuales y estilos de aprendizaje que podrían desencadenar una inercia interesante en el grupo.

Dado que la operación de un curso en este caso se lleva en un medio virtual, será indispensable que el tutor tenga habilidad para comunicarse a través de la escritura. También se deberá tomar en cuenta qué es importante considerar al momento de establecer comunicación con los alumnos: ¿es conveniente que me extienda o no en el mensaje? ¿Elegir el "Asunto" ("Subject”) será importante? ¿Tendré que ser conciso? ¿La cordialidad forma parte del mensaje? ¿Deberé cuidar la veracidad de lo que informo? ¿Podré comunicar todo lo que desee o deberé cuidar de enviar solo lo realmente importante?, etc.

En lo que respecta a la educación en línea son considerados como problemas “... la deserción, la falta de motivación y un sentimiento de soledad en los estudiantes..." (Hernández, 2007, p. 49). Estas situaciones pueden disminuir considerablemente con una oportuna, eficaz, efectiva, eficiente y constante comunicación.

Es muy importante que la comunicación sea bilateral y de respeto, eso fomentará un ambiente de confianza entre alumno y maestro. De acuerdo a Bañuelos y Barrón existen aspectos que “...no sólo se ubican como irrenunciables, sino que avanzan con mayor claridad en el diseño de nuevas formas de organización educativa, como el diálogo, la comunicación, el involucramiento y la identificación con el interlocutor" (2005, p. 45). Es por ello que al proceso de comunicación que se da entre los diferentes actores en el aula virtual, se le identifica como "el elemento fundamental que relaciona y transforma a estos mismos participantes. Es decir, la comunicación les da sentido y dirección a los instrumentos de gestión del conocimiento" (Bañuelos y Barron, 2005, p. 45). 
Una parte esencial de la comunicación es la relacionada con transmitir emociones pues eso será uno de los factores que permita mantener motivados a los alumnos.

Dominio de contenidos: el profesor en esta modalidad se convierte en “... un evaluador permanente del aprendizaje de cada uno de sus estudiantes. Analiza y valora las ejecuciones para consolidar o reorientar los logros" (Quesada, 2006, p. 2).

Para lograr lo anterior en tiempo y forma, una de las variables en juego es el dominio del contenido temático, si no sucediera así podría provocar en el alumno: ansiedad, molestia y un impacto aún más crítico como lo es que el alumno construya en la falsedad. Otro efecto importante, a raíz de esta carencia, podría ser la deserción del curso de parte del alumno e incluso del programa en el que estuviera inscrito. Por este motivo el tutor podría verse también como una figura de la que depende en gran medida, la retención de un alumno.

Informar de las políticas de tiempos de respuesta y respetarlas: hacer eso al inicio del curso disminuirá niveles de ansiedad en el alumno y no permitirá que este cree falsas expectativas sobre los tiempos de respuesta. Cumplir con los tiempos es parte del compromiso del profesor y, el alumno debe tener claro que en una modalidad en línea, el concepto "sincronía" prácticamente desaparece del proceso enseñanza - aprendizaje (Angulo, 2009).

Claridad en la tarea: sin duda la descripción precisa de cada actividad es un factor que aumenta las posibilidades de éxito al entregarla como producto para su evaluación: a través de qué medio se enviará, cuál es la fecha de entrega, qué formato deberá tener, extensión y cualquier otro tipo de especificación requerida. Sin embargo, otro elemento importante que permite evidenciar puntualmente al alumno qué se espera de él es la rúbrica, la cual deberá estar incluida en el diseño de un curso y para cada actividad. La rúbrica será el recurso en el que se base el tutor para llevar a cabo la evaluación de cada actividad, en ella se describirá: qué elementos deberán incluirse, cuántos puntos corresponden a cada uno de ellos, niveles de desempeño y dominio que el estudiante puede reflejar en su actividad.

Conocimiento de la plataforma, otras tecnologías y aplicaciones utilizadas en el proceso: en un modelo de esta naturaleza es indispensable que el tutor tenga el conocimiento mínimo requerido para orientar al alumno ante cualquier situación que se presentara, y además, el contacto de apoyo tecnológico por si la duda estuviera fuera de su alcance. De esta manera podrá apoyarlo en el momento que este requiere y no será ese un obstáculo para que el alumno pueda 
continuar desempeñándose en el curso. Es deseable que parte del perfil del tutor en línea sea la apertura a nuevas tecnologías.

El rol del profesor (tutor): el rol del profesor cambia, de ser un transmisor de contenidos (educación tradicional) a ser un moderador y evaluador constante que promueve la reflexión, el sentido crítico que se autorregula y participa activamente. Promoviendo así, un constante reto intelectual en el alumno.

Promover la autonomía en el alumno: una educación bajo este modelo implica que el alumno sea el protagonista de su aprendizaje, motivo por el cual deberá llegar a ser autodirigido y autorregulado. Entre otras cosas, deberá conocer la página del curso y saber en dónde encontrar cada área o sección y será su responsabilidad estar pendiente tanto de la agenda del curso en la que se mencionan las fechas de realización para cada actividad como de la realización de sus actividades en tiempo $\mathrm{y}$ forma.

Algunas recomendaciones para el tutor en actividades grupales (foros):

- Crear los equipos.

- Crear los espacios adecuados y con la estructura pertinente para cada foro.

- Incluir las indicaciones de la actividad grupal en cada foro.

- Indicar bajo qué técnica didáctica se trabajará y recordar los lineamientos correspondientes.

- Reiterar en el foro, como parte de las indicaciones, qué se espera de ellos como equipo en esa actividad e incluir la rúbrica o direccionarlos a ella.

- Incluir preguntas detonadoras para propiciar la discusión.

- Ofrecer sustento teórico como recurso importante (lecturas).

- Enviar (o solicitar que el equipo la genere) una lista de lo que se evaluará durante la discusión, por ejemplo: calidad de las aportaciones, mínimo de aportaciones, etc.

- Detectar a los alumnos que no hayan ingresado a participar e invitarlos a hacerlo siempre y cuando aún tengan forma de ponerse al corriente permitiéndoles así, seguir con la dinámica de la actividad. 
- Moderar cada foro teniendo siempre en mente: las políticas, reglas, lineamientos, objetivo de la actividad y técnica didáctica que se esté utilizando.

- Solicitar que cada aportación, inicial y posteriores, se justifiquen en las lecturas correspondientes.

- Mantener una participación activa y con enfoque reflexivo. El seguimiento debe ser continuo, así el tutor podrá seguir conduciendo o bien reconduciendo el aprendizaje y, de esta forma verificar que el conocimiento se vaya construyendo con veracidad.

- Mantener un clima adecuado para cada equipo (respeto, trabajo, justicia, cordialidad, apertura, etc.) e intervenir oportunamente cuando así se requiera.

- Evitar dar respuestas directas a preguntas que se le hagan y más bien, llevar a los alumnos a que ellos mismos descubran las respuestas.

- Provocar interacción entre los miembros de cada equipo para que sigan construyendo.

- Mantener comunicación con el equipo como tal y motivarlos.

- Evaluar de una manera justa la participación de cada miembro del grupo, basándose en rúbricas y lineamientos previamente dados a conocer a los alumnos.

- Generar y compartir con los participantes del foro una síntesis del trabajo realizado en su foro con las ideas clave y que condujeron al objetivo de la misma. Podría también generarse una general que reflejara el trabajo de todos y compartirla con todo el grupo.

- Promover el uso de herramientas como coevaluación y autoevaluación en este tipo de actividad.

Un seguimiento como este permitirá que el estudiante que no hubiera participado se dé cuenta que el profesor lo detectó y se preocupó por él y como consecuencia, la actividad en el foro podría aumentar debido a que los alumnos sabrán que su maestro está pendiente de cada cosa que sucede en ese espacio. 
Este tipo de actividades grupales en ambientes virtuales ofrece al tutor la posibilidad de dar un seguimiento detallado al proceso de construcción completo, logrando ver todas y cada una de las interacciones en cada equipo, cosa que no sucede en un contexto presencial. De esta forma, no solo recibirá un producto final para su evaluación sino que el tutor será capaz de estar realizando una evaluación constante mediante el flujo de comunicación que se va dando en cada equipo. Los foros virtuales son una gran fortaleza para una educación en línea y es precisamente ahí, en donde el tutor evidencia la calidad de su labor y su liderazgo intelectual.

Al aprendizaje colaborativo se le reconoce por ser un “...proceso socioconstructivo que favorece la educación a partir de un trabajo conjunto que se traduce en comunidades de aprendizaje donde todos sus miembros aprenden "de y con" los otros, bajo los principios de diversidad, tolerancia, empatía, colaboración y cooperación; además de la negociación, el diálogo y la toma de decisiones consensuadas que facilitan múltiples respuestas ante una misma situación en contextos diferentes en los que éstos se produjeron" (Iglesias, 2012, p.461). Lo anterior promueve un aprendizaje que se enriquece cada vez que los miembros del equipo participan provocando así, que se genere un conocimiento holístico.

La riqueza del aprendizaje que se genera en un medio virtual depende directamente de la capacidad, experiencia y conocimiento de los integrantes del grupo que interactúan a través del diseño instruccional del curso, permaneciendo como actores dinámicos en los diferentes escenarios (Chan, 2005).

En este mismo concepto de foros, se dice que el ambiente propicio para generar aprendizaje es aquel que deja espacios abiertos a profesores y alumnos que facilitan su interacción provocando así, aprendizaje significativo (Chan, 2005).

La actividad en un foro “...favorece el pensamiento crítico y las relaciones interpersonales entre sus participantes, el trabajo autónomo, la motivación, la discusión y/o contraste de opiniones, la redacción y expresión escritas, el intercambio de experiencias y la eliminación de barreras tanto temporales como espaciales, entre otras..." (Iglesias, 2012, p. 466).

La importancia de la interacción y comunicación en un medio virtual exige nuestra atención hacia un franco desarrollo de ellas entre los participantes en el grupo, pues es precisamente la interacción la que “...no solo promueve la evaluación entre alumno y profesor sino también entre pares” (Mogollón, 2004). La interacción detona además los procesos de construcción del conocimiento y formación académica. 
Es importante también tener en mente algunas situaciones no deseables que se pueden presentar para estar conscientes y, como tutores, saber cómo manejarlas: sentimiento de aislamiento de parte del alumno si no recibiera respuesta rápida a sus aportaciones; ausencia de comunicación verbal; dificultad para detectar el fin de una conversación; incremento significativo en el tiempo dedicado al seguimiento de parte del profesor y posible sobrecarga de información para el alumno (Iglesias, 2012).

"Las tecnologías apoyan y facilitan el aprendizaje constructivista mientras los alumnos trabajan en línea puesto que... los alumnos adquieren conocimiento y construyen sus habilidades sobre la base de actividades y experiencias compartidas con otros alumnos... De esta manera, el alumno desarrolla algunas de las competencias clave en Internet: la colaboración, la negociación, la reflexión, la crítica constructiva, la selección y análisis de la información" (Grodecka, Pata y Väljataga, 2008, p.5. En: Iglesias, 2012, p. 464).

\section{Actividades individuales}

\section{El profesor deberá:}

- Notificar la recepción del documento.

- Considerar la rúbrica con la que se evaluará y la descripción de la actividad.

- Especificar cuántos puntos pierde en cada apartado de la rúbrica y por qué.

- Mencionar no solo las áreas de oportunidad sino también los aciertos y ajustar lo correspondiente para reforzar el aprendizaje.

- Hacer comentarios, aportaciones y compartir recursos al respecto.

- Provocar retos intelectuales, haciendo preguntas en el contenido de su trabajo en situaciones que lo propicien, eso seguirá generando conocimiento.

- Manejar de manera asertiva los mensajes que acompañen a la retroalimentación.

- Enviar retroalimentación cualitativa y cuantitativa en los tiempos establecidos. 


\section{El alumno deberá:}

- Seguir las indicaciones para la realización de la actividad al pie de la letra.

- Consultar la rúbrica de la actividad, la descripción de la actividad y trabajar en base a ellas.

- Estar pendiente de la retroalimentación que envíe su profesor.

- Revisar a detalle la retroalimentación y considerarla para futuras actividades, aun cuando la calificación fuera satisfactoria para él.

- Enviar dudas al tutor sobre su retroalimentación, si las tuviera.

En cualquier actividad, después de notificar la evaluación y enviar la retroalimentación correspondiente, será importante que el tutor informe al grupo sobre la liberación de la misma y mantenga la apertura para recibir observaciones, dudas e inquietudes al respecto. Lo anterior dará la oportunidad de resolverlas y por qué no, de darse cuenta si hubo algún error al evaluarlo, recordemos la importancia del feedback bilateral.

Cuando el alumno siente la presencia del profesor, aún en una educación en línea, se sentirá arropado, acompañado, motivado y respetado por su profesor.

\section{Otras recomendaciones:}

Cerrar casos: asegurarse de cerrar los casos específicos que se presentaran (revisiones de actividades, quejas, solicitudes, inquietudes, etc.) y conservar evidencias, disminuirá considerablemente casos críticos. No hacerlo mantendrá situaciones críticas latentes que al resurgir en el futuro aumentará la dificultad para resolverlas o desencadenará problemas más severos.

Actitud y sensibilidad: cuando un alumno ha logrado depositar la confianza en su profesor, este deberá responder a ella apoyándolo con criterios establecidos y respetando siempre las reglas, lineamientos y políticas del curso y la institución.

Debe haber comprensión, sin dejar de ser justos con cada uno de los alumnos del grupo, promoviendo siempre el respeto, la disciplina y el orden. 
Mantener la calidez en una modalidad de este tipo no implica romper las reglas. Recordemos además que el lenguaje juega un papel preponderante y eso incluye la expresión emocional que en los mensajes se transmite.

El maestro, con rol ahora de moderador, deberá mantener una comunicación oportuna, adecuada, efectiva y eficiente; deberá ser comprometido y autodirigido, de tal forma que, a diario revise su curso.

Considerar lo recomendado en este artículo le permitirá al profesor un adecuado seguimiento durante el proceso de enseñanza aprendizaje. Se puede decir entonces que un maestro que se desempeña bajo esta modalidad: "a) juega un papel directivo y como guía que varía según los tiempos del proceso educativo; b) es un mediador de los saberes socioculturales; c) planea la actividad cooperativa de los estudiantes; d) es un conocedor de la cognición de sus alumnos; y e) es un experto en su asignatura" (Hernández, 2007, p. 60).

El concepto de retroalimentación, después de lo expuesto a través de este documento, podría definirse como: un producto resultante de la revisión y el análisis por parte del profesor a la actividad, aportación o proyecto que ha enviado el (los) estudiante(s). En ese producto se deben reflejar: observaciones, correcciones, aportaciones, reforzamiento y una evaluación cuantitativa basada en una rúbrica y, la puntual descripción de la actividad. El profesor envía esta retroalimentación al (los) alumno (s) de manera oportuna, permitiéndole(s) así conocer aciertos, áreas de oportunidad y aspectos precisos que deberá ajustar para seguir construyendo con veracidad su conocimiento y enriqueciéndolo. Una retroalimentación de calidad deberá ser: cualitativa, cuantitativa y oportuna.

\section{CONCLUSIÓN}

En la actualidad la educación en línea ha venido a revolucionar los procesos de enseñanza aprendizaje, esto es: pedagogía soportada en tecnología.

"Una visión sistémica de las circunstancias y procesos involucrados en la instrumentación exitosa de la educación en línea implica reconocer su complejidad, junto con su potencial y su versatilidad, que nos invitan a respetar la diversidad, a buscar las diferencias y similitudes, los aciertos y errores, a aprender pero también a desaprender" (McAnally y Organista, 2007, p. 93).

La educación en línea logra vislumbrarse como una solución para educar a grandes masas y, dada su naturaleza promueve el desarrollo de habilidades y 
competencias muy particulares, que no se dan en otro formato. Como resultado del gran auge que ha demostrado tener la modalidad, capta la atención para que se sigan desarrollando nuevas formas que brinden calidad educativa en el proceso.

La retroalimentación es fundamental, pues es a través de ésta que el alumno no solo se da cuenta de sus fortalezas y debilidades, sino que es una oportunidad que el profesor tiene para promover la reflexión en el aprendizaje y construir en un continuo durante todo el curso y generar así, un conocimiento aún más profundo y significativo.

Para llevar a cabo el proceso de retroalimentación con resultados satisfactorios es importante considerar que son tres las variables que influyen: "las habilidades pedagógicas del tutor, el nivel de actividad de los alumnos y un esfuerzo compartido entre ambas partes" (Jackson y Graesser, 2006).

Recordemos además, que para que la retroalimentación esté completa debe incluir tres elementos: "Feed - U", "Feed - Back" y "Feed - Forward"; hacia dónde voy, cómo me está yendo y qué sigue ahora.

La comunicación es parte esencial dentro del proceso de retroalimentación, Jensy y Solano, mencionan que “...es muy importante brindar asesoramiento personalizado al estudiantado, motivarlos, darles recomendaciones públicas y privadas sobre los trabajos y sobre su progreso en los estudios" (2010, p. 6).

La entrega a una labor como esta se refleja en el actuar de un maestro que no pierde de vista su vocación, la cual se finca en "...la ética del docente y el compromiso con la educación de los individuos” (Román, 2009, p. 12).

Siendo la calidad lo que se está buscando y la retroalimentación la columna vertebral del tutoreo se logra ver a esta última como aquella que “...permite constatar los logros de los estudiantes y promover el aprendizaje con información de retorno, constituyéndose en una herramienta de control de calidad" (Mogollón, 2004, p. 44).

\section{REFERENCIAS BIBLIOGRÁFICAS}

Angulo Hernández, L. M. (2009). Proyecto: educación en línea. Revista Electrónica Educare, VIII (1). (123-133). [en línea] Disponible en: http://www.redalyc.org/ articulo.oa?id=194114416009 (consulta 2013, 6 de junio).
Bañuelos Márquez, A. M.; Barrón Soto, H. (2005). Modelos para la gestión del conocimiento para la educación en línea. Universidad de Guadalajara, 5 (1), (44-53). [en línea] Disponible en: http://www.redalyc.org/articulo. 
oa?id=68850105 (consulta 2013, 6 de junio).

Barberá, E. (2006). Aportaciones de la tecnología a la e-Evaluación. Revista de Educación a Distancia, V (VI), (1-13). [en línea] Disponible en: http://www. $\underline{\text { redalyc.org/articulo.oa?id }=54709901}$ (consulta 2013, 28 de junio).

Campos Céspedes, J.; Brenes Matarrita, O. L.; Solano Castro, A. (2010). Competencias del docente de educación superior en línea. Revista Electrónica Actualidades Investigativas en Educación, 10 (3), (1-19). [en línea] Disponible en: http://www.redalyc.org/articulo. oa?id=44717980010 (consulta 2013, 6 de junio).

CIMED (2005). El poder transformador de la educación. Innovación Educativa, 5 (27), (55-58). [en línea] Disponible en: http://www.redalyc.org/articulo. oa?id=179421423006 (consulta 2013, 6 de junio).

Chan Núñez, M. E. (2005). Competencias mediacionales para la educación en línea. Revista Electrónica de Investigación Educativa, 7 (2), (1-21). [en línea] Disponible en: http://www. redalyc.org/articulo.oa?id=15507209 (consulta 2013, 6 de junio).

Hattie J.; Timperley H. (2007). The Power of Feedback. Review of Educational Research, 77 (1), (81-112). [en línea] Disponible en: http://rer.sagepub.com/ content/77/1/81.abstract (consulta 2013, 1 de julio).

Hernández Gallardo, S. C. (2007). El constructivismo social como apoyo en el aprendizaje en línea. Universidad de Guadalajara, 7 (7), (46-62). [en línea] Disponible en: http://www.redalyc. org/articulo.oa?id=68800705 (consulta 2013, 6 de junio).

Iglesias Rodríguez, A. (2012). Feedback y feed forward a través de los foros. Experiencia en un curso online de la Universidad de
Salamanca. Teoría de la Educación. Educación y Cultura en la Sociedad de la Información, 13 (1), (459-477). [en línea] Disponible en: http://www.redalyc.org/ articulo.oa?id=201024387016 (consulta 2013, 28 de junio).

Jackson, G.; Graesser, A. (2006). Aplicaciones del diálogo humano de tutoría al Auto Tutor: un sistema inteligente de tutoría. Revista Signos, 39 (60), (31-48). [en línea] Disponible en: http://www.redalyc.org/articulo. oa? $\mathrm{id}=157013768002$ (consulta 2013, 28 de junio).

McAnally Salas, L.; Organista Sandoval, J. (2007). La educación en línea y la capacidad de innovación y cambio de las instituciones de educación. Universidad de Guadalajara, 7 (7), (82-94). [en línea] Disponible en: http://www.redalyc. org/articulo.oa?id=68800707 (consulta 2013, 28 de junio).

Mogollón, I. (2004). El chat y otros procedimientos de evaluación a distancia aplicables en sistemas mixtos. Revista de Medios y Educación, 23, (43-54). [en línea] Disponible en: http://www. redalyc.org/articulo.oa?id $=36802304$ (consulta 2013, 28 de junio).

Quesada Castillo, R. (2006). Evaluación del aprendizaje en la educación a distancia "en línea“. Revista de Educación a Distancia, $V$ (VI) (1-15). [en línea] Disponible en: http://www.redalyc. org/articulo.oa?id=54709902 (consulta 2013, 6 de junio).

Román Maldonado, C. E. (2009). Sobre la retroalimentación o el feedback en la educación superior on line. Revista Virtual Universidad Católica del Norte, 26, (1-18). [en línea] Disponible en: http://www.redalyc.org/articulo. oa?id=194215516009 (consulta 2013, 6 de junio). 


\section{PERFIL ACADÉMICO Y PROFESIONAL DE LA AUTORA}

Marta Araceli Alvarado García. Miembro del equipo fundador de Universidad TecMilenio y del programa "Carreras por Internet" del Sistema Tecnológico de Monterrey. En los últimos 14 años se ha desempeñado en la modalidad en línea en el Sistema Tecnológico de Monterrey a cargo de los siguientes roles: diseñadora de cursos, profesora, coordinadora y directora del programa de profesional, diseñadora de planes de estudio, directora académica de profesores y actualmente como maestra de cátedra en la Escuela de Graduados en Educación del mismo sistema.

E-mail: maalvara@tecvirtual.mx

DIRECCIÓN POSTAL DE LA AUTORA

Universidad TecVirtual del Sistema Tecnológico de Monterrey

Ave. Eugenio Garza Sada 2501 Sur,

Col. Tecnológico

C.P. 64849 , Monterrey, N.L., México

Fecha de recepción del artículo: $02 / 10 / 13$

Fecha de aceptación del artículo: 16/12/14

\section{Como citar este artículo:}

Alvarado García, M. A. (2014). Retroalimentación en educación en línea: una estrategia para la construcción del conocimiento. RIED. Revista Iberoamericana de Educación a Distancia, volumen 17, $\mathrm{n}^{\mathrm{O}}$ 2, pp. 59-73. 